\title{
Identification of Endogenous SUM01 Accepter Sites by Mass Spectrometry
}

\author{
He-Hsuan Hsiao, Erik Meulmeester, and Henning Urlaub
}

\begin{abstract}
Posttranslational modification (PTM) by the covalent conjugation of small ubiquitin-like modifier (SUMO) plays an important role in many biological processes, such as cell cycle progression, transcriptional regulation, subcellular transport, and other processes. An in-depth understanding of the function of SUMOylation requires the discovery of SUMO accepter sites. However, identification of endogenous SUMO-conjugated sites in higher eukaryotes by MS-based proteomic strategies is hampered by the low abundance of SUMO conjugates, the large tryptic fragments of SUMOI or SUMO2/3 and the inability to match MS/MS spectra by protein database search engine. In this chapter, we describe a powerful method to overcome at least some of these challenges. To identify SUMO acceptor sites in endogenous SUMOI conjugated protein, the SUMOI conjugates are purified by immunoprecipitation with anti-SUMOI antibodies followed by SDS-PAGE separation and in-gel tryptic digestion. The resulting peptides are either performed using standard data dependent acquisition (DDA) for protein identification or high mass DDA to enhance the sensitivity of detection on the LTQ-Orbitrap mass spectrometer. Finally, a Web-based database tool, ChopNSpice, coupled with a protein database search engine is introduced to ease the identification of SUMOI attachment sites. Although this method was initially used to identify SUMOI accepter sites, it can be readily adapted to study SUMO2/3 conjugates or even other Ubiquitin-like modifiers.
\end{abstract}

Key words: ChopNSpice, Liquid chromatography, Mass spectrometry, Posttranslational modification, Proteomics, Small ubiquitin-like modifier

\section{Introduction}

The ability to identify posttranslational modifications (PTMs) of proteins and to exactly localize the sites of attachment is a critical step for understanding the biological meaning of modifications. Recently, mass spectrometry (MS) has emerged as a powerful technology for protein mapping studies, peptide and protein sequence 
analysis, and identification of PTMs in proteins (for summary see Chapter 7), including phosphorylation (see Chapters 21 and 22), glycosylation (see Chapter 23), oxidation (see Chapter 24), nitrosylation (see Chapter 25) acetylation, ubiquitylation (see Chapter 26) and SUMOylation (1-4). However, the enormous complexity of the proteome and the large dynamic range of protein expression impede the analysis of PTMs by MS. The small ubiquitin-like modifier (SUMO) proteins are members of the ubiquitin-like family of proteins that are reversible posttranslational modifiers. Covalent conjugation of SUMO to its targets requires the attachment of the C-terminal glycine residue of SUMO to the $\varepsilon$-amino group of a lysine residue in the target protein. The modification of the target by SUMO subsequently regulates protein-protein interaction, or intra-molecular interactions, emerging as an important regulatory mechanism in a diverse range of cellular processes (5-9).

Global proteomic approaches to identify novel SUMO substrates from yeast Saccharomyces cerevisiae have begun to emerge in recent years (10-13). These studies generally rely upon affinity purification of SUMOylated proteins from yeast S. cerevisiae, followed by the identification of SUMO accepter sites using mass spectrometric techniques. The presence of amino acid sequence (-EQIGG) on the modified lysine residue after digestion of the SUMO conjugates by trypsin leads to a 484-Da increase in the mass of the peptide that can be readily measured by mass spectrometry. The MS/MS spectra of these SUMOconjugated peptides can be identified by a protein database search that considers for this mass shift (484 Da) on the modified lysine residue. Unfortunately, such strategies are difficult to be applied for the identification of SUMO accepter sites in mammalians due to the larger distance of Arg or Lys residue to the $\mathrm{C}$ terminus of mammalian SUMO-1, -2, and -3. As a consequence, the large SUMO-conjugated peptides from mammalians that are generated after trypsin digestion impede their identification by database searches. Identification of such large conjugated peptides using MS/MS spectra is only possible as the peptides of substrates within the protein database are also modified by SUMO sequence. ChopNSpice (4) is a powerful software tool to automatically generate SUMO-conjugated sequences of proteins in silico that is compatible with current proteomics search engines such as Mascot (14) or SEQUEST (15) (see Chapter 28) for the identification of SUMO accepter sites.

In this chapter, we describe a method for identifying SUMOylation sites isolated from HeLa-S3 cells using LTQOrbitrap mass spectrometry. The general workflow for globally mapping human endogenous SUMOI accepter sites is depicted in Fig. 1. Briefly, the purification of human SUMOl conjugates is carried out by an immunoprecipitation with $\alpha$-SUMOl antibodies 


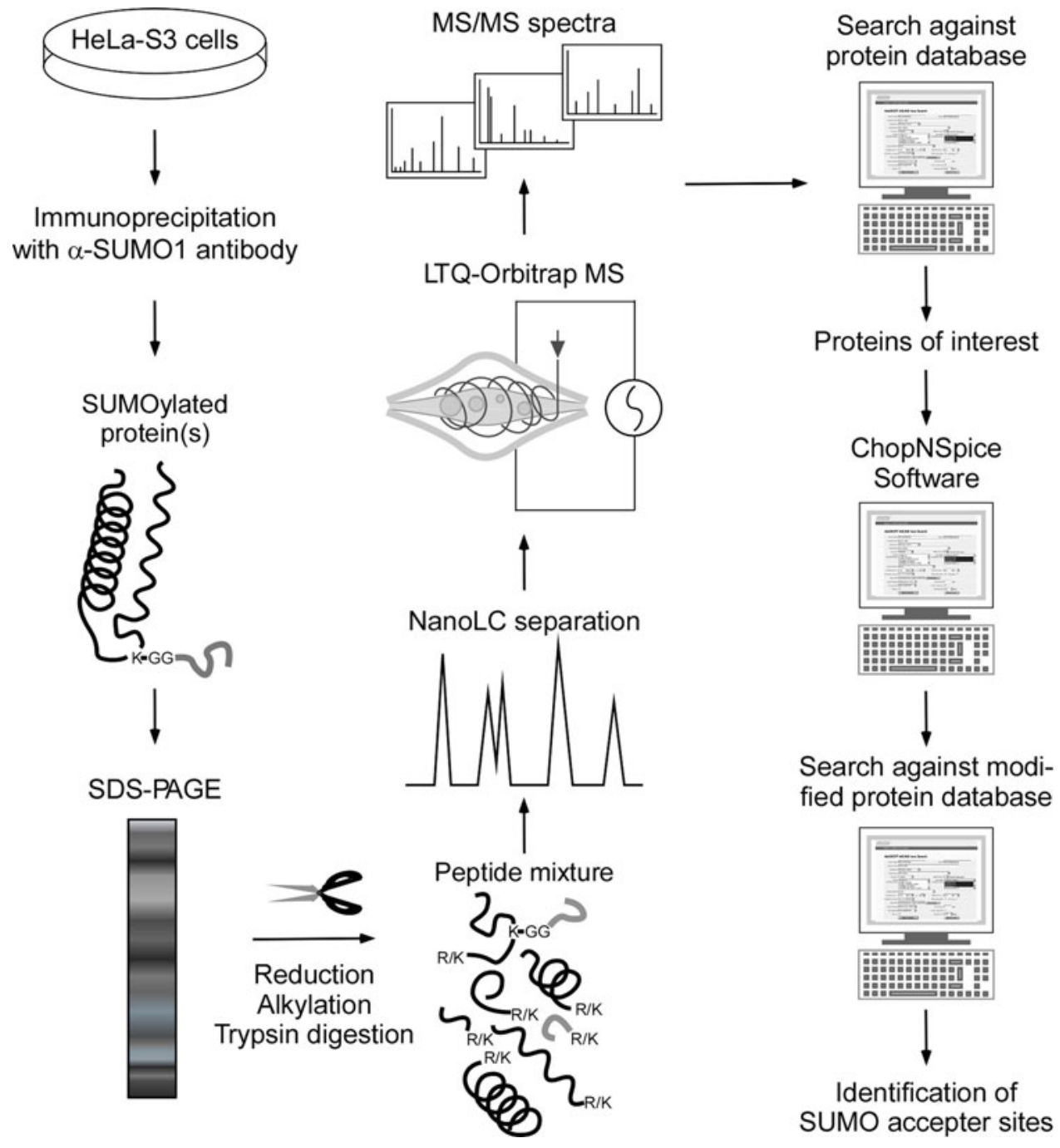

Fig. 1. Strategy for mapping endogenous human SUM01 attachment sites.

from HeLa-S3 cells. The SUMOylated proteins are then separated by SDS-PAGE with Coomassie blue staining and cut equally into 20 slices. Each slice is reduced, alkylated, and subsequently digested with trypsin. Half of tryptic peptides are separated by reversephase nanoLC chromatography and detected with LTQ-Orbitrap mass spectrometer with standard data dependent acquisition (DDA). The resulting MS/MS spectra are searched against for protein identification. The proteins of interest found in the individual bands are performed a second run of MS/MS acquisition under high mass conditions, where only those precursor ions exceeding the mass of the SUMOl C-terminal tryptic fragment $(2,154 \mathrm{Da})$ are selected for sequencing. Both the MS/MS analyses 
are merged into single peak list file for further protein database search. The proteins of interest are put into ChopNSpice software to create virtual SUMOylated protein sequences. The merging peak list file is resubmitted to search against modified protein database for identification of SUMO-conjugated sites.

\section{Materials}

\subsection{Immuno- precipitation of SUM01 Conjugates}

1. Joklik's medium (Sigma-Aldrich, St. Louis, MO).

2. Fetal bovine serum (Invitrogen, Carlsbad, CA).

3. Antibiotics: Penicillin or Streptomycin stock solution (Merck, Darmstadt, Germany).

4. PBS: store at $4^{\circ} \mathrm{C}$.

5. N-ethylmaleimide (NEM, Sigma-Aldrich).

6. TB: $10 \mathrm{mM}$ PIPES, $55 \mathrm{mM}$ manganese chloride $\left(\mathrm{MnCl}_{2}\right)$, $15 \mathrm{mM}$ calcium chloride $\left(\mathrm{CaCl}_{2}\right), 250 \mathrm{mM}$ potassium chloride $(\mathrm{KCl})$.

7. Triton X-100 (Merck).

8. Adenosine triphosphate (ATP, Sigma-Aldrich).

9. Protease inhibitors (Sigma-Aldrich).

10. $0.45-\mu \mathrm{m}$ filter (Millipore, Billerica, MA).

11. Monoclonal $\alpha$-SUMO1 antibodies (GMP1, Zymed, South San Francisco, CA).

12. Protein G-agarose (GE Healthcare, Chalfont, UK).

13. Washing buffer: $20 \mathrm{mM} \mathrm{NaP}$ ( $\mathrm{pH} 7.4$ ), $150 \mathrm{mM} \mathrm{NaCl}, 1 \%$ Triton, $0.5 \%$ sodium deoxycholate, $0.1 \%$ SDS, 10 mM NEM.

14. Elution buffer: $2 \times$ SDS sample buffer.

1. Ultrapure water $\left(\right.$ LiChrosolv $\left.^{\circledR}\right)$.

2. Acetonitrile $(\mathrm{ACN})\left(\mathrm{LiChrosolv}^{\circledR}\right)$.

3. Ammonium bicarbonate (ABC) buffer: $25 \mathrm{mM} \mathrm{ABC} \mathrm{(Sigma-}$ Aldrich), $\mathrm{pH}$ 8.5.

4. Dithiothreitol (DTT) solution: $25 \mathrm{mM}$ DTT in $25 \mathrm{mM} \mathrm{ABC}$, $\mathrm{pH}$ 8.5. Prepared freshly before use.

5. Iodoacetamide (IAA) solution: $50 \mathrm{mM}$ IAA in $25 \mathrm{mM} \mathrm{ABC}$, $\mathrm{pH}$ 8.5. Prepared freshly before use (see Note 1).

6. Trypsin stock solution: $0.1 \mu \mathrm{g} / \mu \mathrm{L}$ sequencing-grade endoprotease trypsin (Promega Corp., Madison, WI, sequencing grade) in $25 \mathrm{mM}$ ABC, $\mathrm{pH} 8.5$.

7. Extraction buffer: $50 \%$ ACN, $5 \%$ trifluoroacetic acid (TFA). 


\subsection{Determination of SUM01 Conjugates Using NanoLC Coupled with Mass Spectrometry}

1. Agilent 1100 HPLC system (Agilent Technologies, Santa Clara, CA).

2. Linear Ion Trap-Orbitrap mass spectrometer integrated with a nanoelectrospray ion source (LTQ-Orbitrap MS, Thermo Fisher Scientific, Waltham, MA).

3. HPLC mobile phase A (loading buffer): $0.1 \%$ formic acid (FA) in water.

4. HPLC mobile phase B: $0.1 \%$ formic acid and $95 \% \mathrm{ACN}$ in water.

5. Cl8 trap column: $150 \mu \mathrm{m} \times 1.5 \mathrm{~cm}, 5 \mu \mathrm{m}$ Cl8-AQ resin (Dr. Maisch GmbH, Ammerbuch-Entringen, Germany) in $360 \mu \mathrm{m}$ O.D. fused silica capillary.

6. C18 analytical column: $75 \mu \mathrm{m} \times 10 \mathrm{~cm}, 5 \mu \mathrm{m} \mathrm{Cl8}-\mathrm{AQ}$ resin (Dr. Maisch $\mathrm{GmbH}$ ) in $360 \mu \mathrm{m}$ O.D. fused silica emitter capillary.

1. Software to convert MS/MS spectra from the raw data to peaklist file (mgf file) using BioworksBrowser 3.3.1 SPI (Thermo Fisher Scientific).

2. Software to identify SUMO conjugates, such as Mascot 2.2.07 (Matrix Science, London, UK).

3. Software, ChopNSpice, to automatically generate SUMOconjugated sequences of proteins in silico (freely available online http://chopnspice.gwdg.de).

\section{Methods}

\subsection{General Practice}

To identify the modification for the ubiquitin-like proteins of interest requires the purification of the entire pool of conjugates, in our case SUMOI, from the cell line of choice. Subsequently the conjugates are separated by SDS-PAGE, digested with trypsin, the peptide components of that mixture are analyzed using nanoLCOrbitrap mass spectrometry and finally the SUMOl-conjugated sites are identified using the Mascot search engine integrated with ChopNSpice software.

1. Maintain HeLa-S3 cells in Joklik's medium containing 10\% fetal bovine serum and antibiotics.

2. Wash HeLa-S3 cells $\left(1 \times 10^{9}\right)$ twice with PBS containing $10 \mathrm{mM}$ NEM.

3. Lyse in 2 pellet volumes of TB containing $0.1 \%$ Triton and $10 \mathrm{mM}$ ATP supplemented with protease inhibitors and $10 \mathrm{mM}$ NEM. 


\subsection{Tryptic Digest of SUM01 Conjugates and Extraction of SUM01- Conjugated Peptides}

4. Centrifuge $16,000 \times g$ for $15 \mathrm{~min}$ at $4^{\circ} \mathrm{C}$.

5. Filter through a $0.45-\mu \mathrm{m}$ filter.

6. Incubate the supernatant with $100 \mu \mathrm{g}$ of monoclonal $\alpha$-SUMOl antibodies (GMPl) for $2 \mathrm{~h}$ at $4^{\circ} \mathrm{C}$.

7. Centrifuge $16,000 \times g$ for $15 \mathrm{~min}$ at $4^{\circ} \mathrm{C}$.

8. Incubate the supernatant with protein $\mathrm{G}$-agarose resins for $2 \mathrm{~h}$ at $4^{\circ} \mathrm{C}$.

9. Wash the resins four times with $750 \mu \mathrm{L}$ of washing buffer.

10. Elute bound proteins with $30 \mu \mathrm{L}$ of elution buffer.

11. Separate the eluted proteins by SDS-PAGE followed by Coomassie blue staining (see Note 2 and Chapter 4 ).

1. Excise entire lane from SDS-PAGE into 20 gel slices (see Note 3).

2. Cut each slice into pieces of $1 \times 1 \mathrm{~mm}$ using a scalpel and a spatula and transfer these pieces into $0.5-\mathrm{ml}$ Eppendorf tube for tryptic digestion.

3. Wash the gel pieces with $400 \mu \mathrm{L} \mathrm{ABC}$, spin the gel pieces down and remove all liquid.

4. Add $400 \mu \mathrm{L}$ ACN to shrink the gel pieces, spin the gel pieces down and remove all liquid.

5. Add $60 \mu \mathrm{L}$ DDT solution to reduce the disulfides and incubate in a thermomixer for $1 \mathrm{~h}$ at $37^{\circ} \mathrm{C}$.

6. Spin the gel pieces down and remove all liquid.

7. Add $400 \mu \mathrm{L}$ ACN to shrink the gel pieces, spin the gel pieces down and remove all liquid.

8. Add $60 \mu \mathrm{L}$ IAA solution to alkylate the reduced cysteines and incubate in a thermomixer for $1 \mathrm{~h}$ at $37^{\circ} \mathrm{C}$.

9. Repeat steps 6 and 7.

10. Add $60 \mu \mathrm{L}$ trypsin solution $(0.01 \mu \mathrm{g} / \mu \mathrm{L})$ to digest proteins and incubate in a thermomixer at least for $16 \mathrm{~h}$ at $37^{\circ} \mathrm{C}$ (see Note 4).

11. Add $100 \mu \mathrm{L}$ ACN to shrink the gel pieces, spin the gel pieces down and transfer all liquid to the other $0.5-\mathrm{ml}$ Eppendorf tube.

12. Add $60 \mu \mathrm{L}$ extraction buffer, vortex for $10 \mathrm{~s}$, sonicate for $1 \mathrm{~min}$, spin the gel pieces down, and transfer all liquid to the corresponding Eppendorf tube.

13. Repeat step 12 three times.

14. Add $60 \mu \mathrm{L}$ ACN to shrink the gel pieces, spin the gel pieces down and transfer all liquid to the corresponding Eppendorf tube.

15. Repeat step 14. 
16. Dry down the supernatant with a SpeedVac.

17. Store the dried sample at $-20^{\circ} \mathrm{C}$ for further MS analysis.

\subsection{Determination of SUM01 Conjugates Using NanoLC Coupled with Mass \\ Spectrometry}

\subsubsection{Nano Liquid \\ Chromatography}

3.4.2. Mass Spectrometry
All NanoLC-MS/MS analyses were performed on a LTQ-Orbitrap XL mass spectrometer equipped with a nanoLC ESI source, connected to an Agilent 1100 LC system with an autosampler under the software control of Xcalibur 2.0.7.

1. Dissolve the tryptic peptides with $4 \mu \mathrm{L}$ of $0.1 \%$ FA in $50 \%$ $\mathrm{ACN}$, vortex for $10 \mathrm{~s}$ and sonicate for $5 \mathrm{~min}$ (see Note 5 ).

2. Add $16 \mu \mathrm{L}$ of $0.1 \% \mathrm{FA}$, vortex for $10 \mathrm{~s}$ and sonicate for 5 min.

3. NanoLC conditions: Following autosampler injection, the gradient was first held at $100 \%$ mobile phase A for $5 \mathrm{~min}$, raised a 0.5 min linear gradient from 0 to $7.5 \%$ mobile phase $\mathrm{B}$, a $37 \mathrm{~min}$ linear gradient from 7.5 to $37.5 \%$ mobile phase $\mathrm{B}$, a $0.5 \mathrm{~min}$ linear gradient from 37.5 to $80 \%$ mobile phase B, 7 min at $80 \%$ mobile phase $\mathrm{B}$, a 0.5 min linear gradient to $100 \%$ mobile phase $\mathrm{A}$ and finally held at $100 \%$ mobile phase A for $9.5 \mathrm{~min}$.

In this step, parts of tryptic peptides separated by nanoLC are introduced into LTQ-Orbitrap MS through nanoelectrospray ion source. For protein identification, the eluted peptide ions are analyzed in a standard data-dependent acquisition. First, the $\mathrm{m} / \mathrm{z}$ values of the peptides are detected and the most five intense ions are selected for collision-induced dissociation (CID) MS/MS, in which a peptide is fragmented in turn. The MS/MS spectra contain the peptide sequence information and are used to search protein database for protein identification. In addition, the remains of tryptic peptides are performed with high mass data-dependent acquisition. Under this condition, only peptides with a mass exceeding SUMO are triggered for MS/MS acquisition. This approach is highly sensitive for the detection and sequencing of larger SUMO conjugated-peptides.

1. Operate the LTQ-Orbitrap MS in the standard data-dependent acquisition to automatically trigger MS/MS scan by using the Tune and Xcalibur 2.0.7. software.

2. Set the following parameters in the Tune page:
(a) Spray voltage at $1.8 \mathrm{kV}$.
(b) Heated capillary temperature at $150^{\circ} \mathrm{C}$.
(c) Accumulation target value at $1 \times 10^{6}$ and $1 \times 10^{4}$ for MS and MS/MS scan, respectively.
(d) Maximum injection time at $500 \mathrm{~ms}$ and $250 \mathrm{~ms}$ for MS and MS/MS scan, respectively. 
3. Set the following parameters in the Xcalibur software:

(a) A lock-mass ion from ambient air at $\mathrm{m} / \mathrm{z} 445.120025$ for internal calibration.

(b) Full scan MS in the $\mathrm{m} / \mathrm{z}$ range from 350 to 2,000 with a resolution $R=30,000$ at $\mathrm{m} / \mathrm{z} 400$.

(c) Five most intense multiply charged ions to be measured in the MS/MS scan.

(d) Set the threshold for MS/MS scan at 1,000 counts.

(e) Set normalized collision-induced dissociation (CID) collision energy at $37.5 \%$ for MS/MS in LTQ.

(f) An activation $q=0.25$.

(g) Activation time $=30 \mathrm{~ms}$.

(h) Dynamic exclusion of up to 500 precursor ions for $60 \mathrm{~s}$ upon MS/MS scan and exclusion mass width of $10 \mathrm{ppm}$.

4. For high mass data-dependent acquisition, the settings are identical to standard data-dependent acquisition in addition to the followed parameters in the Xcalibur software.

(a) Set an additional three microscans in LTQ MS/MS scan.

(b) The mass range for selecting MS-data-dependent masses was set 2,154-1,000,000 for SUMOl-conjugated peptides, and the option of $\mathrm{m} / \mathrm{z}$ value as masses had to be enabled (see Note 6).

3.5. Data Analysis and Identification of SUMOylation Site
Peak list files (mgf format) are generated from MS raw data by Bioworks browser, containing precursor masses and their corresponding product ion masses and intensities. The mgf file is used to match peptide sequence information by the Mascot search engine for protein identification. The proteins of interest are put into ChopNSpice software to create a new protein database. The search is repeated against the modified protein database and the SUMO acceptor sites can be identified.

1. Convert MS/MS spectra from the raw LC/MS/MS datafile to single mgf file using Bioworks browser (see Note 7).

2. Submit mgf file to Mascot search engine for protein identification with the following parameters: trypsin specificity; allow up to two missed cleavages; consider methionine oxidation and cysteine carboxyamidomethylation as variable modifications; instrument setting ESI-TRAP; mass tolerance of $10 \mathrm{ppm}$ in MS mode and 0.8 Da in MS/MS mode.

3. The sequence of the protein of interest was manually saved as a Fasta file. 
4. Submit the protein Fasta file to ChopNSpice software (http:// chopnspice.gwdg.de) to create a new Fasta file with the following parameters:

(a) Spice species: h. sapiens.

(b) Spice: SUMOl.

(c) Spice site: KX.

(d) Spice mode: once per fragment.

(e) Included unmodified fragments in output: yes.

(f) Enzyme: Trypsin-N-term K or R, not C-term P.

(g) Protein miscleavages min: 0 .

(h) Protein miscleavages max: 3.

(i) Miscleavages in spice: $0-1$.

(j) Included minor end fragments: yes.

(k) Output formatting: FASTA : Single protein sequence.

(1) Cleavage marker: Mark all cleaved sites ("J").

(m) Retain comments in FASTA format: yes.

(n) Line breaks in FASTA output: yes.

5. Resubmit mgf file to Mascot search engine against a new Fasta file generated by ChopNSpice for identification of SUMOIconjugated sites with the following parameters: enzyme cleaved at $\mathrm{J}$ at $\mathrm{N}$ - and $\mathrm{C}$ terminus; allow zero missed cleavages; consider methionine oxidation and cysteine carboxyamidomethylation as variable modifications; instrument setting ESI-TRAP; mass tolerance of $10 \mathrm{ppm}$ in MS mode and 0.8 Da in MS/MS mode (see Note 8).

\section{Notes}

1. IAA is light sensitive and has to be stored in the dark after preparation.

2. A small aliquot of SUMOI conjugates may be analyzed at this stage by SDS-PAGE followed by immunoblotting with $\alpha$-SUMOl antibodies (GMPl) to monitor the effectiveness of the purification.

3. Cutting the whole lane ensures that the entire sample will be analyzed.

4. The optimum trypsin amount is highly dependent on protein quantity. Experimenter has to adjust the amount accordingly.

5. The potential problem for analysis SUMOI conjugates by MS arises from the adhesion between Eppendorf tube and for large 
SUMOl-conjugated peptides. High percentage of organic solvent and sonication are essential to redissolve those large peptides.

6. Increase of additional microscans can improve the quality of MS/MS spectrum for mapping SUMOl-conjugated sites via database searching and high mass data-dependent acquisition enhance the sensitivity to identify the acceptor sites from more complex sample.

7. Mascot does not support to search MS/MS fragment ions with a charge state higher than 2, resulting in larger SUMO1conjugated peptides with charge state of +4 or +5 show a low Mascot score or are not identified at all. MS/MS spectra recorded in the FT analyzer/detector of the Orbitrap MS with sufficient resolution for charge-state recognition have to use software tool Raw2msn (16) to deconvolute the higher charge stages of the fragment ions in the raw data to singly charged fragment ions for Mascot search that in turn increase the identification.

8. A new enzyme allowed cutting $\mathrm{J}$ at both $\mathrm{C}$ and $\mathrm{N}$ terminus have to be created in Mascot server. The file "quant_subs.pl" in the folder (mascot $|c g i|$ ) must be changed from $J \geq 0$ to $J \geq 0.05$ in line 3653. In addition, SUMO-conjugated sites also can be identified by using other search engines e.g., SEQUEST, $\mathrm{X}$ ! Tandem, etc.

\section{References}

1. Mann M, Ong SE, Gronborg M et al (2002) Analysis of protein phosphorylation using mass spectrometry: deciphering the phosphoproteome. Trends Biotechnol 20:261-268

2. Kim SC, Sprung R, Chen Y et al (2006) Substrate and functional diversity of lysine acetylation revealed by a proteomics survey. Mol Cell 23:607-618

3. Meierhofer D, Wang X, Huang L, Kaiser P (2008) Quantitative analysis of global ubiquitination in HeLa cells by mass spectrometry. J Proteome Res 7:4566-4576

4. Hsiao HH, Meulmeester E, Frank BT et al (2009) "ChopNSpice," a mass spectrometric approach that allows identification of endogenous small ubiquitin-like modifier-conjugated peptides. Mol Cell Proteomics 8: 2664-2675

5. Seeler JS, Dejean A (2003) Nuclear and unclear functions of SUMO. Nat Rev Mol Cell Biol $4: 690-699$
6. Johnson ES (2004) Protein modification by SUMO. Annu Rev Biochem 73:355-382

7. Hay RT (2005) SUMO: a history of modification. Mol Cell 18:1-12

8. Geiss-Friedlander R, Melchior F (2007) Concepts in sumoylation: a decade on. Nat Rev Mol Cell Biol 8:947-956

9. Meulmeester E, Melchior F (2008) Cell biology: SUMO. Nature 452:709-711

10. Panse VG, Hardeland U, Werner T et al (2004) A proteome-wide approach identifies sumoylated substrate proteins in yeast. J Biol Chem 279:41346-41351

11. Denison C, Rudner AD, Gerber SA et al (2005) A proteomic strategy for gaining insights into protein sumoylation in yeast. Mol Cell Proteomics 4:246-254

12. Wohlschlegel JA, Johnson ES, Reed SI, Yates JR 3rd (2004) Global analysis of protein sumoylation in Saccharomyces cerevisiae. J Biol Chem 279:45662-45668 
13. Hannich JT, Lewis A, Kroetz MB et al (2005) Defining the SUMO-modified proteome by multiple approaches in Saccharomyces cerevisiae. J Biol Chem 280:4102-4110

14. Perkins DN, Pappin DJ, Creasy DM, Cottrell JS (1999) Probability-based protein identification by searching sequence databases using mass spectrometry data. Electrophoresis 20:3551-3567
15. Eng JK, Mccormack AL, Yates JR (1994) An approach to correlate tandem mass-spectral data of peptides with amino-acid-sequences in a protein database. J Am Soc Mass Spectrom 5:976-989

16. Olsen JV, de Godoy LM, Li G et al (2005) Parts per million mass accuracy on an Orbitrap mass spectrometer via lock mass injection into a C-trap. Mol Cell Proteomics 4:2010-2021 